\title{
Parasitic (Helminthic) Infection While on Asthma Biologic Treatment: Not Everything Is What It Seems
}

This article was published in the following Dove Press journal:

Journal of Asthma and Allergy

\author{
Laren D Tan $\mathbb{D}^{1,2, *}$ \\ Brett Schaeffer $\mathbb{D}^{3, *}$ \\ Abdullah Alismail (iD ${ }^{2}$ \\ 'Division of Pulmonary, Critical Care, \\ Hyperbaric, Allergy and Sleep Medicine, \\ Loma Linda University Health, Loma \\ Linda, California, USA; ${ }^{2}$ Department of \\ Cardiopulmonary Sciences, School of \\ Allied Health Professions, Loma Linda \\ University, Loma Linda, California, USA; \\ ${ }^{3}$ Department of Internal Medicine, \\ School of Medicine, Loma Linda \\ University, Loma Linda, California, USA \\ *These authors contributed equally to \\ this work
}

Correspondence: Laren D Tan Department of Medicine, Division of Pulmonary, Critical Care, Hyperbaric, Allergy and Sleep Medicine, Loma Linda University School of Medicine, II 234

Anderson Street, Suite 6439, Loma Linda, California 92354, USA

Tel + I 909558808 I

Fax + I909558058|

Email LaTan@llu.edu

\begin{abstract}
Asthma is a chronic inflammatory disorder of the airway that is characterized by bronchial hyperresponsiveness and variable airflow limitation. Approximately 235 million people are affected by asthma worldwide and $5-10 \%$ are considered to be refractory to standard asthma treatment. These patients are known to have repeated exacerbations requiring multiple courses of systemic corticosteroids and as a result, are at risk for increased adverse effects (i.e., osteoporosis, infections). Several new medications known as biologic agents have been approved for the treatment of moderate-to-severe asthmatics. These biologic agents target essential parts of the cell-mediated allergic and to a lesser degree nonallergic immune response (IgE, IL-5, and IL-4/IL-13). They are gaining more favor in the treatment of moderate-to-severe asthma due to their efficacy and excellent safety profile. Despite the most common adverse events being minor, such as injection site reactions, upper respiratory infections, or headaches, these agents carry a small risk of more severe complications such as anaphylaxis and decreased defense against parasitic infections (PI). The incidence of PI compared with other rare adverse events is not well reported, and there are no consensus guidelines for risk prevention of PI in asthmatics undergoing evaluation for, or currently using, biologic therapy. Thus, this article sets out to review the incidence of reported PI and other rare adverse events among asthmatics using current FDA-approved biologic therapies. Secondly, we discuss the clinical implications for the importance of risk prevention of PI with the use of biologic therapies in asthmatics. Lastly, we share an educational handout to assist providers in informing their patients of behaviors that could potentially increase their risk of PI while being on a biologic agent.
\end{abstract}

Keywords: asthma, biologics, parasitic infection, $\operatorname{IgE}$

\section{Introduction}

Asthma is a prevalent reactive airway disease in developed countries and is increasingly growing in developing countries. ${ }^{1}$ The last decade has seen a bright turn in the development and use of biologic therapies for severe asthma management. ${ }^{2}$ There are currently five Food and Drug Administration (FDA)-approved biologic therapies for the management of severe allergic or eosinophilic asthma: omalizumab, mepolizumab, reslizumab, benralizumab, and dupilumab (Figure 1). In addition to these approved therapies, there are also several new biologics currently under investigational study for severe asthma treatment. ${ }^{3}$ The available biologic therapy can be clustered into three categories, those that target immunoglobulin E (IgE) (omalizumab), interleukin-5 (IL-5) (reslizumab, benralizumb, and mepolizumab), and interleukin-4 and 13 (IL-4)/ (IL-13) (dupilumab). Biologic therapy has also been included in the most recent Global Initiative for Asthma (GINA) 2019 guidelines. $^{4}$ Like all medications, biologic therapies have side effects and 


\section{Omalizumab}

2003
Reslizumab

2016

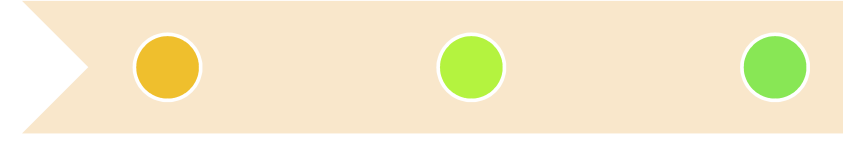

2015
Mepolizumab
Dupilumab

2018

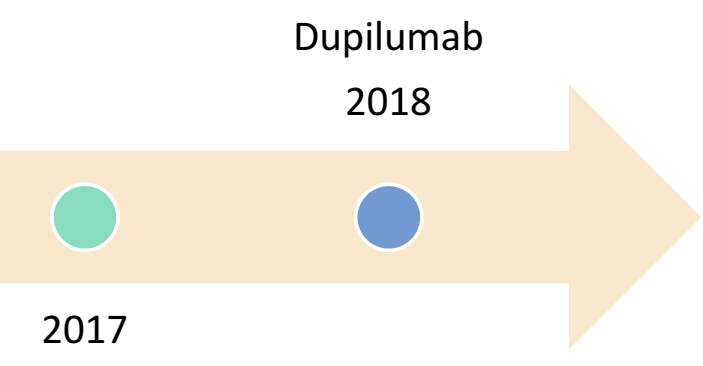

2017

Figure I Food and Drug Administration (FDA) approved biologic therapy for severe asthma. Note: The following references include the documents for the FDA drug approval details. ${ }^{24-28}$

adverse events that health-care providers and patients must be aware of when using these therapies. A rare, and under-studied adverse event that can occur with biologic therapy use is the acquisition of a parasite, more specifically a helminthic infection. ${ }^{5-9} \operatorname{IgE}$ and eosinophils play an important role in a host's defense against parasites. Therefore, therapies that target IgE, or cytokines involved in the allergen response pathway, can theoretically lead to an increased risk of acquiring or not being able to clear parasites. ${ }^{9}$ Because of its rarity and the limited long-term data we have on the newer biologics, the amount of discussion of parasites during the selection process for biologic therapy is unknown amongst clinicians. To the author's knowledge, aside from reviewing the everyday activities that make up the patient's life also known as life-markers, there is no standardized clinical practice guideline for clinicians and patients to review specific factors or behaviors that could potentially increase their risk in acquiring a parasite while on biologic therapy. On the other hand, rare outcomes such as anaphylaxis and hypereosinophilia currently have implementations in place to prevent or reduce the risk of occurrence. ${ }^{10}$ Patients are provided epi-pens in the event of anaphylactic reaction, and patients are also tested for eosinophil levels prior to starting therapy, in order to check for hypereosinophilia. ${ }^{11}$ This, in fact, raises the question of should we not also provide patients with education on how to prevent parasitic infection while on biologic therapy? This paper will serve as a review of the current literature on biologic therapy regarding their relationship with the immune system and parasites, and the incidence of parasitic infection and other adverse events. It also includes a link to a patient-centered educational handout that lists the high-risk behaviors associated with parasitic infections.

\section{Methods}

An advanced search on PubMed, Dynamed, and Google Scholar was done for selecting articles related to this topic using the following keywords: "Omalizumab", "Mepolizumab", "Reslizumab", "Benralizumab", "Dupilumab", "Asthma", "Parasitic Infection", and "Anaphylaxis". In addition, data were gathered from drug trials that were performed on all the FDA-approved biologics to check for any reported incidences.

\section{Pathophysiology of the Immune System's Defense Against Parasites in an Asthmatic} The pathogenesis of asthma involves allergens binding to antigen-presenting cells and subsequently binding to $T$ helper (Th) cells, which then differentiate into $\mathrm{T}$ helper $2\left(\mathrm{~T}_{\mathrm{H}} 2\right)$ cells and secrete key inflammatory drivers IL-4, IL-5, and IL-13. These cytokines then activate B cells to secrete IgE. IL-5 also activates eosinophils, which is part of the pro-inflammatory allergic response in asthma. IgE binds to mast cells at the FCERI receptor, allowing them to recognize the allergen and release an allergic inflammatory reaction and cause smooth muscle bronchoconstriction via histamine, prostaglandins, and leukotrienes. ${ }^{8}$ Asthma occurs explicitly when there is an abnormal development of Th cells into $\mathrm{T}_{\mathrm{H}} 2$ cells leading to increased production of interleukins and $\mathrm{IgE}$ and increased activation of B cells, mast cells, and eosinophils. ${ }^{1}$

Parasitic infections act similarly to allergens that trigger asthma by stimulating a $\mathrm{T}_{\mathrm{H}} 2$ cell response and a cascade of increased interleukins, IgE, mast cells, and eosinophils. In particular, there is a considerable increase in IL-4, IL-10, IL13 , and IgE and a slightly less increase in IL-5 production compared to allergen response. ${ }^{1}$

IgE bound mast cells bind allergen to release further inflammatory response and help remove/destroy the allergen. ${ }^{12}$ Similarly, parasites are destroyed by the same method via IgE bound mast cells and the release of inflammatory cytokines. ${ }^{1}$ 


\section{Pharmacology of Biologic Therapy for Severe Asthma}

Asthma biologic therapies target several of the critical steps involved in this cell-mediated pathway, including anti-IgE (omalizumab), anti-IL-5 (mepolizumab, reslizumab, benralizumab), and anti-IL-4/IL-13 (dupilumab). There are other interleukins, as well as different drugs that target the same pathways as above, which are currently involved in clinical trials. However, those will not be discussed here, because they are currently not FDA approved for treatment in asthma management. ${ }^{2,12}$

\section{Anti-lgE (Omalizumab)}

$\operatorname{IgE}$ is a crucial factor in asthma pathogenesis and leads to mast cell activation and further release of the inflammatory cascade. ${ }^{1}$ Subsequently, it became the first target of biologic therapy with the introduction of omalizumab in $2003 .^{2,12}$ Omalizumab is a monoclonal antibody that binds free $\operatorname{IgE}$ to form IgE-omalizumab complexes which then act as a paratope to free IgE and bind the FceRI and FceRII receptors of mast cells, basophils, and dendritic cells, blocking free IgE from binding. With the IgE-omalizumab complex bound to the FceRI receptor on mast cells, there is no degranulation of inflammatory cytokines. It also downregulates the expression of further FceRI receptors. ${ }^{2,12}$

\section{IL-5 (Mepolizumab, Reslizumab, and Benralizumab)}

When evaluating for severe refractory asthma treatment, blood eosinophil evaluation is becoming a common practice. ${ }^{2}$ According to GINA, ${ }^{4}$ eosinophils $>150$ cells $/ \mu \mathrm{L}$ are considered to be an indicator of elevated type 2 inflammation. Depending on the eosinophilia and severity of asthma, patients could then be initiated on anti-IL-5 biologic therapy. ${ }^{2}$ IL-5 is a proinflammatory cytokine that is released by $\mathrm{T}_{\mathrm{H}} 2$ cells, mast cells, and eosinophils and is also involved in further stimulating activation, proliferation, and differentiation of eosinophils. ${ }^{8}$ Mepolizumab (FDA approved in 2015) and reslizumab (FDA approved in 2016) are monoclonal antibodies that target IL-5 and block it from binding the IL-5 receptor, while Benralizumab (FDA approved in 2017) targets explicitly the IL-5 receptor alpha (IL-5R $\alpha$ ) on eosinophils, mast cells, natural killer cells, and basophils. ${ }^{13,14}$ Benralizumab blocks activation and proliferation of these cells, but also causes antibody-mediated cell cytotoxicity (apoptosis) of eosinophils and basophils, leading to almost complete eradication of these cells. $^{2,12}$ These monoclonal antibody therapies decrease the levels of eosinophils in the blood and airways and lead to reduced inflammation and improvement in asthma symptoms.

\section{IL-4 and IL-I 3 (Dupilumab)}

IL-4 and IL-13 are inflammatory cytokines secreted primarily by $\mathrm{T}_{\mathrm{H}} 2$ cells and act on $\mathrm{B}$ cells to cause IgE isotype switching, $\mathrm{T}_{\mathrm{H}} 2$ cell proliferation and airway changes (i.e. smooth muscle hypertrophy, goblet cell hyperplasia, fibrosis $)^{2,12}$ Dupilumab is a monoclonal antibody to the IL- $4 \alpha$ receptor, which blocks the binding of IL-4 and IL-13. ${ }^{12}$ Dupilmab was first approved for the treatment of refractory moderate to severe atopic dermatitis in 2017 and later approved in 2018 for treatment of asthma in patients over 12 years of age with moderate to severe asthma with an eosinophilic phenotype or those with oral corticosteroid-dependent asthma. ${ }^{15}$

\section{Incidence of Adverse Events}

In 2017, Bagnasco et al (2017) reviewed seven anti-IL-5 and anti-IL-5R clinical trials: one on mepolizumab $(n=1460)$, two clinic trials on reslizumab $(n=530)$, and five clinical trials on benralizumab $(n=2138) .{ }^{16}$ They reported two cases of anaphylaxis with reslizumab and no anaphylaxis with mepolizumab or benralizumab or any parasitic infections reported with all three drugs. The COLUMBA study (2019) is the longest follow-up study for the safety of patients with severe eosinophilic asthma receiving Mepolizumab and there were no reported parasitic infections among enrolled patients. ${ }^{17,18}$ Moreover, eight RCTs and clinical trials on omalizumab with a combined total of 1966 patients receiving therapy had four cases of anaphylaxis and one case of a gastrointestinal parasite. $5,19,20$

Two reviews on data of patients using omalizumab $(n=57,300$ and $n=39,510)$ between 2003 and 2006 found an anaphylaxis rate of $0.09-0.2 \%$ (Table 1$){ }^{11,21}$ Only one RCT has been published so far in regard to patients at high risk for parasite infection, whereas the previous trials were mostly performed in modernized countries. The trial was performed on individuals in Brazil with high risk for parasites who were given omalizumab for asthma. Results showed a slight increased risk for acquiring parasitic infection when compared with placebo (50\% vs $41 \%$ ); however, the sample size was small and the results were not statistically significant $(\mathrm{P}=0.14){ }^{7}$ RCTs on antiIL-4/IL-13 therapy such as dupilumab have also shown no occurrences of parasites or anaphylaxis; however, another rare adverse event, hypereosinophilia, occurred between $0.14 \%$ and $4.1 \%$ of the cases. ${ }^{10}$ 
Table I Incidence of Rare Adverse Events (Parasitic Infection, Anaphylaxis, and Hypereosinophilia) Among Food and Drug Administration Approved Biologic Agents

\begin{tabular}{|l|l|l|l|}
\hline Therapy & $\begin{array}{l}\text { Incidence } \\
\text { of } \\
\text { Parasite }\end{array}$ & $\begin{array}{l}\text { Incidence of } \\
\text { Anaphylaxis }\end{array}$ & $\begin{array}{l}\text { Incidence of } \\
\text { Hypereosinophilia }\end{array}$ \\
\hline Omalizumab & $0.05 \%^{\mathrm{a}}$ & $0.09-0.20 \%$ & None reported \\
\hline Mepolizumab & $\begin{array}{l}\text { None } \\
\text { reported }\end{array}$ & $\begin{array}{l}\text { None } \\
\text { reported }\end{array}$ & None reported \\
\hline Reslizumab & $\begin{array}{l}\text { None } \\
\text { reported }\end{array}$ & $0.38 \%$ & None reported \\
\hline Benralizumab & $\begin{array}{l}\text { None } \\
\text { reported }\end{array}$ & $\begin{array}{l}\text { None } \\
\text { reported }\end{array}$ & None reported \\
\hline Dupilumab & $\begin{array}{l}\text { None } \\
\text { reported }\end{array}$ & $\begin{array}{l}\text { None } \\
\text { reported }\end{array}$ & $0.14-4.1 \%$ \\
\hline
\end{tabular}

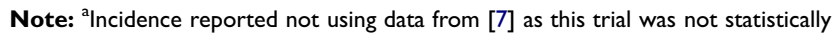
significant and specifically targeted patients at high risk for parasite. Data from references $5,10,16,19,20$, and 29-31.

\section{Clinical Considerations and Implications}

Despite these adverse events being rare, patients are still screened for blood eosinophil levels and excluded not to receive certain biologic therapies (omalizumab or dupilumab) if their blood eosinophil level is too high ( $>300$ cells/ $\mu \mathrm{L}),{ }^{2,8}$ and patients who do qualify to undergo therapy are given an epi-pen to treat anaphylaxis if it were to occur. ${ }^{11}$ The Asthma and Allergy Joint Commission investigated anaphylaxis as an adverse event of omalizumab therapy and created recommended guidelines for clinicians prescribing it - explicitly including the most common timeframe of anaphylaxis occurrences, long-term monitoring, and epi-pen prescriptions. ${ }^{11}$ If these rare adverse events have enough significance to require screening tests and the distribution of epi-pens, then it is reasonable to include parasitic infection risk in the conversation as well. Like the guidelines created by the Joint Commission, education for most common types of parasites, how to acquire them, risk factors, and signs/symptoms could be provided to clinicians to give to patients qualifying for therapy.

The proposed rationale behind an increased risk of parasitic infection while on biologic therapy is as follows. When $\mathrm{IgE}$ and the inflammatory cytokines (IL-4, IL-5, and IL-13) are inhibited, there is a decreased response to an allergen, lower number of eosinophils, and an overall improvement in asthma and its symptoms. ${ }^{12}$ On the other hand, the inability to respond appropriately to allergens can lead to some adverse events that biologic therapy display. In particular, IgE and eosinophils are essential parts of the body's immune response to parasitic infections. IgE and eosinophils allow the body to directly kill and eliminate the parasites, and without them or the cytokines involved in the pathway, the expulsion of parasitic infections becomes limited. ${ }^{9}$ This has been tested in several mouse models where genes for IL-4/IL-13 were removed, leading to a decrease in host response to parasitic infection. ${ }^{9}$ Interestingly, mouse models performed with removal of IgE showed no inhibition or expulsion of Schistosoma and decreased burden of Strongyloides. ${ }^{22,23}$ As a result, there is a mixed picture of how biotherapy would affect humans with parasites or their risk of acquiring a parasite; therefore, many randomized control trials (RCTs) and clinical trials performed on biologic therapy in humans excluded patients with parasites or who had traveled to countries with a high prevalence of parasitic infection. ${ }^{9}$

Although long-term real-world studies are still lacking on asthmatic patients infected with helminths while on biologics, and as a result definitive guidelines in relation to the prevention of infection by helminths cannot be provided. Therefore, clinicians should still be sure to obtain a thorough history and ask about the current and past history of parasitic disease, travel history, and contact with others who may have been exposed to or currently have a parasite prior to starting biologic therapy. Patients on biologic therapy are at risk for parasitic infection and should be educated on the high-risk behaviors that could result in a parasitic infection. To better meet this knowledge gap, an educational sheet was created to educate and remind patients of these high-risk behaviors (see high-risk sheet handout). This sheet could also be embedded electronically to remind prescribers to assess these high-risk behaviors before starting biologic therapy.

\section{Conclusion}

Inflammatory cytokines involved in the cell-mediated allergic response are interesting targets of modern biologic therapy for the treatment of moderate-to-severe asthma. These therapies are not without their adverse risk and events, despite serious events being rare, they are still noteworthy and require thorough clinician and patient understanding prior to initiating therapy. The role of IgE and allergic response cytokines in a host's defense against parasites has been previously studied in mouse models with results showing a mixed, undetermined relationship. ${ }^{9}$ There is also a lack of enough human trials on biologic therapy and its relationship to parasitic infection to create a conclusion on its long-term effect. The only trial completed focusing on this relationship showed a possible 
connection to an increased rate of parasitic infection. ${ }^{7}$ With a lack of definitive evidence to disprove that biological therapy increases the risk of parasitic infections, it is crucial that healthcare providers are thoroughly discussing this topic and its risks. The biologic high-risk behavior handout should be provided to all asthmatic patients who are being considered or currently on biologic therapy. It is important that more studies be completed regarding biologic therapy and its relationship to parasites, especially in underdeveloped countries undergoing economic transition with access to biologic therapy. Future research should continue to look for a relationship between parasites and these medications, as well as study the incidence of occurrence among low risk vs high-risk individuals. The idea that reduction in IgE could play a role in improving the expulsion of parasites is an exciting topic for future research and possibly a further role for biologic therapy. ${ }^{22,23}$

\section{Acknowledgment}

We would like to extend our gratitude and thanks to Dr. Bradley E. Chipps, MD FAAP, FACAAI, FAAAAI, FCCP, for his time to review our paper.

\section{Disclosure}

Laren D Tan is a speaker/consultant for AstraZeneca, Boehringer Ingelheim, Regeneron, Novartis, Sanofi Genzyme, and Boston Scientific; reports personal fees from Boehringer Ingelheim, Boston Scientific, AstraZeneca, Sanofi Genzyme, and Regeneron, outside the submitted work; and reports no other conflicts of interest in this work. The other authors report no conflicts of interest, sponsorships, or funding to disclose for this article.

\section{References}

1. Sitcharungsi R, Sirivichayakul C. Allergic diseases and helminth infections. Pathog Glob Health. 2013;107(3):110-115. doi:10.1179/ 2047773213Y.0000000080

2. Menzella F, Galeone C, Bertolini F, Castagnetti C, Facciolongo N. Innovative treatments for severe refractory asthma: how to choose the right option for the right patient? J Asthma Allergy. 2017;10:237. doi:10.2147/JAA.S144100

3. Manka LA, Wechsler ME. Selecting the right biologic for your patients with severe asthma. Ann Allergy Asthma Immunol. 2018;121 (4):406-13.

4. Global Initiative for Asthma. Pocket Guide for Asthma Management and Prevention: A Pocket Guide for Health Professionals; updated 2019. Available from: https://ginasthma.org/wp-content/uploads/2019/04/ GINA-2019-main-Pocket-Guide-wms.pdf. Accessed 25 November, 2019.

5. Yalcin AD, Bisgin A, Cetinkaya R, Yildirim M, Gorczynski RM. Clinical course and side effects of anti-IgE monoclonal antibody in patients with severe persistent asthma. Clin Lab. 2013;59(1-2):71-77. doi:10.7754/Clin.Lab.2012.120406
6. Cooper PJ, Ayre G, Martin C, Rizzo JA, Ponte EV, Cruz AA. Geohelminth infections: a review of the role of $\operatorname{IgE}$ and assessment of potential risks of anti-IgE treatment. Allergy. 2008;63(4):409-417. doi:10.1111/all.2008.63.issue-4

7. Cruz A, Lima F, Sarinho E, et al. Safety of anti-immunoglobulin E therapy with omalizumab in allergic patients at risk of geohelminth infection. Clin Exp Allergy. 2007;37(2):197-207. doi:10.1111/ cea.2007.37.issue-2

8. Lanier B Unanswered clinical questions and speculation about the role of anti-immunoglobulin E in atopic and nonatopic disease. Paper Presented At: Allergy \& Asthma Proceedings. 2006.

9. Braddock M, Hanania NA, Sharafkhaneh A, Colice G, Carlsson M. Potential risks related to modulating interleukin-13 and interleukin-4 signalling: a systematic review. Drug Safety. 2018;41(5):489-509. doi:10.1007/s40264-017-0636-9

10. Castro M, Corren J, Pavord ID, et al. Dupilumab efficacy and safety in moderate-to-severe uncontrolled asthma. $N$ Engl J Med. 2018;378 (26):2486-2496. doi:10.1056/NEJMoa1804092

11. Cox L, Platts-Mills TA, Finegold I, Schwartz LB, Simons FER, Wallace DV. American academy of allergy, asthma \& immunology/ American college of allergy, asthma and immunology joint task force report on omalizumab-associated anaphylaxis. $J$ Allergy Clin Immunol. 2007;120(6):1373-1377. doi:10.1016/j.jaci.2007.09.032

12. McCracken J, Tripple J, Calhoun WJ. Biologic therapy in the management of asthma. Curr Opin Allergy Clin Immunol. 2016;16 (4):375. doi:10.1097/ACI.0000000000000284

13. Tan LD, Bratt JM, Godor D, Louie S, Kenyon NJ. Benralizumab: a unique IL-5 inhibitor for severe asthma. J Asthma Allergy. 2016;9:71-81. doi:10.2147/JAA.S78049

14. Benralizumab (FASENRA) Reference ID: 4125440. Published 2016. Available from: https://www.fda.gov/media/110322/download. Accessed May 18, 2019.

15. Administration FaD. Dupilumab: Reference ID: 4337903. 2018.

16. Bagnasco D, Ferrando M, Varricchi G, Puggioni F, Passalacqua G, Canonica GW. Anti-interleukin 5 (IL-5) and IL-5Ra biological drugs: efficacy, safety, and future perspectives in severe eosinophilic asthma. Front Med. 2017;4:135. doi:10.3389/fmed.2017. 00135

17. Khatri S, Moore W, Gibson PG, et al. Assessment of the long-term safety of mepolizumab and durability of clinical response in patients with severe eosinophilic asthma. J Allergy Clin Immunol. 2019;143 (5):1742-1751 e1747. doi:10.1016/j.jaci.2018.09.033

18. Bradford ES, Albers FC, Gilson MJ, et al. Long-term safety of mepolizumab in patients with severe eosinophilic asthma: the COLUMBA study. In: A34 ASTHMA CLINICAL STUDIES I; 2018: A1367-A1367. American Thoracic Society.

19. Busse W, Corren J, Lanier BQ, et al. Omalizumab, anti-IgE recombinant humanized monoclonal antibody, for the treatment of severe allergic asthma. J Allergy Clin Immunol. 2001;108(2):184-190. doi:10.1067/mai.2001.117880

20. Teach SJ, Gill MA, Togias A, et al. Preseasonal treatment with either omalizumab or an inhaled corticosteroid boost to prevent fall asthma exacerbations. J Allergy Clin Immunol. 2015;136(6):1476-1485. doi:10.1016/j.jaci.2015.09.008

21. Corren J, Casale T, Lanier B, Buhl R, Holgate S, Jimenez P. Safety and tolerability of omalizumab. Clin Exp Allergy. 2009;39 (6):788-797. doi:10.1111/cea.2009.39.issue-6

22. Amiri P, Haak-Frendscho M, Robbins K, McKerrow JH, Stewart T, Jardieu P. Anti-immunoglobulin E treatment decreases worm burden and egg production in Schistosoma mansoni-infected normal and interferon gamma knockout mice. J Exp Med. 1994;180(1):43-51. doi:10.1084/jem.180.1.43

23. Korenaga M, Watanabe N, Tada I. Effects of anti-IgE monoclonal antibody on a primary infection of Strongyloides ratti in mice. Parasitol Res. 1991;77(4):362-363. doi:10.1007/BF00930917 
24. Administration USFaD. Novel Drug Approvals for 2016. Food and Drug Administration. Published 2018. Available from: https://www. fda.gov/drugs/new-drugs-fda-cders-new-molecular-entities-and-newtherapeutic-biological-products/novel-drug-approvals-2016. Accessed November 22, 2019

25. Administration USFaD. Novel Drug Approvals for 2017. Food and Drug Administration. Published 2018 Available from: https://www.fda.gov/ drugs/new-drugs-fda-cders-new-molecular-entities-and-new-therapeuticbiological-products/novel-drug-approvals-2017. Accessed June, 2019.

26. Highlights of Prescribing Information: Dupixent. Food and Drug Administration; 2019.

27. Administration USFaD. Clinical Review: Biologics Licensing Application No. 103976 Xolair (Omalizumab). Food and Drug Administration; 2009.
28. Administration USFaD. Novel Drug Approvals for 2015. Food and Drug Administration; 2016.

29. Barranco P, Phillips-Angles E, Dominguez-Ortega J, Quirce S. Dupilumab in the management of moderate-to-severe asthma: the data so far. Ther Clin Risk Manag. 2017;13:1139-1149. doi:10.2147/TCRM

30. Rabe KF, Nair P, Brusselle G, et al. Efficacy and safety of dupilumab in glucocorticoid-dependent severe asthma. N Engl J Med. 2018;378 (26):2475-2485. doi:10.1056/NEJMoa1804093

31. Wenzel S, Castro M, Corren J, et al. Dupilumab efficacy and safety in adults with uncontrolled persistent asthma despite use of medium-tohigh-dose inhaled corticosteroids plus a long-acting $\beta 2$ agonist: a randomised double-blind placebo-controlled pivotal phase $2 \mathrm{~b}$ doseranging trial. The Lancet. 2016;388(10039):31-44. doi:10.1016/ S0140-6736(16)30307-5

\section{Publish your work in this journal}

The Journal of Asthma and Allergy is an international, peer-reviewed open-access journal publishing original research, reports, editorials and commentaries on the following topics: Asthma; Pulmonary physiology; Asthma related clinical health; Clinical immunology and the immunological basis of disease; Pharmacological interventions and new therapies. The manuscript management system is completely online and includes a very quick and fair peer-review system, which is all easy to use. Visit http://www.dovepress.com/testimonials.php to read real quotes from published authors. 